\title{
The Eclipsing Triple System U Ophiuchi Revisited
}

\author{
Luiz Paulo R. Vaz ${ }^{1}$, Johannes Andersen ${ }^{2,3}$ and Antônio Claret ${ }^{3}$ \\ ${ }^{1}$ Departamento de Física, ICEx, UFMG, Brazil \\ email: lpv@fisica.ufmg.br \\ ${ }^{2}$ Niels Bohr Institute, Astronomical Observatory, Copenhagen, Denmark \\ ${ }^{3}$ Nordic Optical Telescope Scientific Association, E-38700 Santa Cruz de La Palma, Spain. \\ email: ja@astro.ku.dk \\ ${ }^{4}$ Instituto de Astrofísica de Andalucía, CSIC, Granada, Spain \\ email: claret@iaa.es
}

\begin{abstract}
We have redetermined the absolute dimensions of the mid B-type eclipsing binary U Oph from new light and radial-velocity curves, accounting for both the apsidal motion and the light-time orbit around the third star. The stars in U Oph have masses of 5.27 and 4.74 $\mathrm{M}_{\odot}( \pm 1.5 \%)$ and are located in the middle of the main-sequence band for an an age of $\sim 50$ Myr. U Oph and three other systems (V760 Sco, MU Cas and DIHer) all have components within $10 \%$ of $5 \mathrm{M}_{\odot}$ and ages below $100 \mathrm{Myr}$; we find significant heavy-element abundance differences between these young nearby stars.
\end{abstract}

Keywords. stars: binaries: close: eclipsing: evolution: individual (U Oph, MU Cas, V760 Sco, DI Her

\section{Introduction}

Accurate empirical stellar masses, radii, and luminosities are fundamental test data for stellar models (Andersen 1991). Good data sets exist for stars below $\sim 4 \mathrm{M}_{\odot}$, but good determinations for higher-mass stars remain few. We have therefore reanalyzed the detached, mid-B type eclipsing binary U Oph from new spectroscopic and photometric data, using improved analysis techniques. Our primary results, including figures, are given in the on-line version of the poster and in the research paper by Vaz et al. (2007).

\section{Observations and Analysis}

New light curves of U Oph in the Strömgren $u v b y \mathrm{H} \beta$ system were obtained with the Danish 0.5-m telescope at ESO, La Silla, Chile, in 1992-94; spectroscopic orbits were determined from high-quality coudé spectrograms from the ESO $1.5 \mathrm{~m}$ telescope. Radial velocities were measured from spectral lines without broad damping wings, which may blend and cause errors in the derived masses (Andersen 1991).

Photometric and spectroscopic orbital elements were determined in a fully consistent analysis, taking into account both the exceptionally short-period apsidal motion $(\sim 21.2 \mathrm{yr})$ and the light-time orbit around a third star $\left(P_{3} \sim 38.4 \mathrm{yr}\right)$. A new combined ephemeris for both orbits was determined from 353 published times of minimum. The Wilson-Devinney (WD) light-curve analysis code was modified to treat apsidal and lighttime orbital motion effects together and used to analyse our uvby light and radial-velocity curves simultaneously.

Figures 1-3 of the poster show our observational data and the differences between our new ephemeris and the previous best determination by Kämper (1986). 


\section{Results}

We find the masses and radii of the components of U Oph to be $5.27 \pm 0.09$ and $4.74 \pm 0.07 \mathrm{M}_{\odot}$, and $3.48 \pm 0.02$ and $3.11 \pm 0.03 \mathrm{R}_{\odot}$, respectively. With $\log g \sim 4.1$, both stars are located near the middle of the main-sequence band. From the colour indices, we find $T_{\text {eff }} \sim 16,000 K$ and derive $\log L / L_{\odot} \sim 2.8$ and a distance around 200 pc (details given in Table 2 of the poster).

We have compared our results for U Oph with stellar evolution models, both the latest series by Claret (2004) and the earlier models (Claret 1995, 1997), as well as with two generations of Padova models (Bressan et al. 1993, Girardi et al. 1996 and Girardi et al. 2000). In each case, we have considered models for both $\mathrm{Z}=0.02$ and $\mathrm{Z}=0.01$ in the diagrams of $\log g$ vs. $\log \left(M / \mathrm{M}_{\odot}\right)$ and $\log T_{\text {eff }}$.

In order to extend the comparison to stars of the same mass but different degrees of evolution within the main sequence, we have identified three other binary systems (MU Cas, DI Her, and V760 Sco) with components within $10 \%$ of $5 \mathrm{M}_{\odot}$ and with accurate data in the literature. Of these stars, DIHer is close to the ZAMS, MU Cas near the TAMS, and V760 Sco intermediate in age between DI Her and U Oph.

We find that the differences between different model calculations for the same chemical composition remain within the observational errors, given the small difference in mass and degree of evolution between the components in each system. When metallicity and age are free parameters, models can be selected that fit each system within the errors, and nothing significant is learned about the models themselves - underscoring that stellar models are best tested in binary systems with very different components and known metal abundances (Andersen 1991). For plausible compositions, the four systems are in the age range 0-100 Myr, with U Oph itself around 40-50 Myr.

The data are, however, accurate enough to show that not all four systems can be fit by models for a single value of $\mathrm{Z}$; a range of about a factor 2 must be invoked. We emphasise that this result is obtained already from the $\log M-\log g$ diagram and is thus independent of uncertain temperature-colour relations. The chemical diversity of these young nearby stars presents a challenge to models of Galactic chemical evolution.

\section{Acknowledgements}

We thank Dr. Claud Lacy for kindly providing his ephemeris code. Support from ESO and the Danish Natural Science Research Council is gratefully acknowledged, as is the financial support of the Brazilian institutions CNPq, CAPES, FAPEMIG and FINEP (to LPRV). This research has made use of the Simbad database, operated at CDS, Strasbourg, France, and of NASA's Astrophysical Data System bibliographic services.

\section{References}

Andersen, J. 1991, A\&GAR 3, 91

Bressan A., Fagotto F., Bertelli G., \& Chiosi C. 1993, A\& $A S$ 100, 647

Claret, A. 1995, A\&SA 109, 441

Claret, A. 1997, $A \& A$ 125, 439

Claret, A. 1998, $A \mathscr{E} A$ 330, 533

Claret, A. 2004, $A \& A$ 242, 919

Girardi L., Bressan A., Chiosi C., Bertelli G., \& Nasi E. 1996, A $\& A S$ 117, 113

Girardi L., Bressan A., Bertelli G., \& Chiosi C. 2000, A\&AS 141, 371

Kämper, B.-C. 1986, Ap\&SS 120, 167

Vaz, L.P.R., Andersen, J., \& Claret, A. 2007, A\&AA, in press (accepted March 29, 2007) 